\title{
Paying attention to reading direction
}

\section{Seta Kazandjian and Sylvie Chokron}

The recent Perspective article by Han and Northoff (Culture-sensitive neural substrates of human cognition: a transcultural neuroimaging approach. Nature Rev. Neurosci. 9, 646-654 (2008) $)^{1}$ on the neuroimaging evidence of transcultural differences in neural substrates of cognitive functions provided an excellent and much-needed spotlight on an area of investigation in cognitive neuroscience that has been largely neglected despite its potential impact on our understanding of the neural substrates of cognition. Han and Northoff demonstrate that the brain and mind are not as universal as once believed; rather, they are shaped by our social cultural experience. Despite the breadth of the studies and cultures presented, crosslanguage differences deserve more mention. In particular, we would like to emphasize the role of written-script and reading direction on neurocognitive networks.

The written-script and thus the reading direction of a language have been found to influence several visuospatial tasks, such as the line bisection task ${ }^{2-5}$, straight-ahead pointing ${ }^{6}$ and apparent-movement perception $^{7,8}$, as well as aesthetic-preference judgements ${ }^{9}$ and the perception of facial affect ${ }^{10}$. Furthermore, reading-direction habits were found to bias the spatial-representation direction of verbal action phrases ${ }^{11,12}$, such that sentence subject and object spatial representations were consistent with the reading direction of the participant's language. In all these studies, right-to-left readers showed biases in the opposite direction or showed no bias at all compared with left-to-right readers.

The above-mentioned cognitive tasks have previously been explained with hemispheric specialization-based hypotheses, which assume a universal culture of cognition. However, the mirror performance of right-to-left readers cannot be explained only in terms of hemispheric specialization. First, from a clinical point of view, regardless of written-language direction, specific left- and right-hemispheric lesions produce equivalent neurological and neuropsychological disorders. Second, the effect of reading direction on visuospatial performance can be simulated just by reversing the subject's scanning direction ${ }^{2}$. There is thus mounting evidence of an interaction between reading direction and brain function, and a cultural hypothesis has been proposed ${ }^{13,14}$. According to this hypothesis, although there are proven functional hemispheric specializations, well-trained behaviours based on culture can either reinforce these biases or otherwise influence them. Reading may have this reinforcing role owing to the specific scanning bias associated with each language.

Considering that there are over 500 million right-to-left readers in the world (for example, Arabic, Farsi, Hebrew and Urdu people), the discussion and further investigation of the cognitive and neural functional differences associated with reading direction is overdue and necessary. Furthermore, with the worldwide accessibility of computers and the internet, bilingualism and bidirectional reading have become more common, suggesting that further inquiry into the neural correlates of bidirectional reading is necessary as well. Although the influence of reading direction is clear, its neuroanatomical substrates have not been investigated as yet. For this, not only do we need to pay closer attention to cultural differences and linguistic factors on a variety of cognitive tasks in both neuropsychological assessments and psychophysical studies, we must also, as Han and Northoff highlight, shift towards the use of neuroimaging to further understand the influence of readingdirection habits on neurocognitive processes.

Seta Kazandjian and Sylvie Chokron are at ERT TREAT Vision, Laboratoire de Psychologie et NeuroCognition, UMR 5105 CNRS-Université Pierre Mendès France, 75019, France.

Sylvie Chokron is also at the Service de Neurologie, Fondation Ophtalmologique Adolphe de Rothschild, 75019, France.

e-mails: seta.kazandjian@upmf-grenoble.fr; chokron@ext.jussieu.fr doi:10.1038/nrn2456-c1

1. Han, S. \& Northoff, G. Culture-sensitive neural substrates of human cognition: a transcultural neuroimaging approach. Nature Rev. Neurosci. 9, 646-654 (2008).

2. Chokron, S. $\&$ Imbert, M. Influence of reading habits on line bisection. Brain Res. Cogn. Brain Res. 1, 219-222 (1993).

3. Chokron, S. \& De Agostini, M. Reading habits and line bisection: a developmental approach. Brain Res. Cogn. Brain Res. 3, 51-58 (1995).

4. Chokron, S., Bernard, J. M. \& Imbert, M. Length representation in normal and neglect subjects with opposite reading habits studied through a line extension task. Cortex 33, 47-64 (1997).

5. Zivotofsky, A. Z. Choosing sides: lateralization in line trisection and quadrisection as a function of reading direction and handedness. Brain Res. Cogn. Brain Res. 20, 206-211 (2004).

6. Kazandjian, S. et al. Egocentric reference in bidirectional readers as measured by the straightahead pointing task. Brain Res. 17 Oct 2008 (doi:10.1016/j.brainres.2008.09.098).

7. Morikawa, K. \& McBeath, M. K. Lateral motion bias associated with reading direction. Vision Res. 32, 1137-1141 (1992)

8. Tse, P. U. \& Cavanagh, P. Chinese and Americans see opposite apparent motions in a Chinese character Cognition 74, B27-B32 (2000).

9. Chokron, S. ¿ De Agostini, M. Reading habits influence aesthetic preference. Brain Res. Cogn. Brain Res. 10, 45-49 (2000).

10. Vaid, J. \& Singh, M. Asymmetries in the perception of facial affect: is there an influence of reading habits? Neuropsychologia 27, 1277-1287 (1989).

11. Maass, A. \& Russo, A. Directional bias in the mental representation of spatial events: nature or culture? Psychol. Sci. 14, 296-301 (2003).

12. Dobel, C., Diesendruck, G. \& Bolte, J. How writing system and age influence spatial representations of actions: a developmental, cross-linguistic study. Psychol. Sci. 18, 487-491 (2007).

13. Paulesu, E. et al. A cultural effect on brain function. Nature Neurosci. 3, 91-96 (2000).

14. Chokron, S. On the origin of free-viewing perceptual asymmetries. Cortex 38, 109-112 (2002). 